\title{
Multipartite entanglement for continuous variables: A quantum teleportation network
}

\author{
P. van Loock and Samuel L. Braunstein \\ Quantum Optics and Information Group, \\ School of Informatics, University of Wales, Bangor LL57 1UT, UK
}

\begin{abstract}
We show that one single-mode squeezed state distributed among $N$ parties using linear optics suffices to produce a truly $N$-partite entangled state for any nonzero squeezing and arbitrarily many parties. From this $N$-partite entangled state, via quadrature measurements of $N-2$ modes, bipartite entanglement between any two of the $N$ parties can be 'distilled', which enables quantum teleportation with an experimentally determinable fidelity better than could be achieved in any classical scheme.

PACS numbers: 03.67.-a, 03.65.Bz, 42.50.Dv
\end{abstract}

Entanglement is seen as an essential ingredient in quantum communication and computation. For example, it enables quantum teleportation which was originally proposed for systems of discrete variables [1]. Later, quantum teleportation was also proposed for continuous variables [2,3]. The simplest teleportation schemes rely on bipartite entanglement, the entanglement of a pair of systems shared by two parties. For pure states, this kind of entanglement is well-understood and can be quantified [4. Multipartite entanglement, the entanglement shared by more than two parties, is much more difficult to quantify [5]. Yet in the laboratory, the creation of tripartite discrete-variable entanglement, yielding so-called GHZ states [6], has been reported for single-photon polarization states [7] and using nuclear magnetic resonance [8].

Continuous-variable quantum teleportation of arbitrary coherent states has been realized experimentally with bipartite entanglement built from two single-mode squeezed vacuum states combined at a beamsplitter [9]. In the absence of entanglement the best mean fidelity of the reconstructed coherent states is $F=\frac{1}{2}$ [10]. Experimentally, $F=0.58 \pm 0.02$ was achieved. Though this limits our attention to the teleportation of a rather modest set of non-orthogonal states, the fidelity gives a clear experimental signal for the presence of entanglement.

Now it is known that even one single-mode squeezed state incident on a beamsplitter yields a bipartite entangled state [11]. This result is in agreement with entropic measures of bipartite pure-state entanglement [12]. If one single-mode squeezed state were distributed among $N$ parties using linear optics would we obtain a truly $N$-partite entangled state? We will show that we can answer this question using the fidelity criterion for teleporting unknown coherent states. In particular, we will see that one single-mode squeezed state is sufficient to allow quantum teleportation between any two of the $N$ parties with the help of all other parties. The assistance by the other $N-2$ parties only relies on local measurements and classical communication. Due to these $N-2$ measurements, bipartite entangled states are 'distilled' from the initial $N$-partite entangled state.

The 'position' and 'momentum' of a 1-D wavepacket (units-free with $\hbar=\frac{1}{2}$ as in Ref. [13]) are the electric quadrature amplitudes representing the quantum state of a single polarization of a single transverse mode of electromagnetic radiation. We define the action of an ideal (phase-free) beamsplitter operation on a pair of modes $i$ and $j$ via

$$
\hat{B}_{i j}(\theta):\left\{\begin{array}{l}
\hat{a}_{i} \rightarrow \hat{a}_{i} \cos \theta+\hat{a}_{j} \sin \theta \\
\hat{a}_{j} \rightarrow \hat{a}_{i} \sin \theta-\hat{a}_{j} \cos \theta
\end{array} .\right.
$$

It yields an entangled state when applied to the zeromomentum eigenstate $|p=0\rangle \propto \int d x|x\rangle$ of mode 1 and the zero-position eigenstate $|x=0\rangle$ of mode 2 :

$$
\hat{B}_{12}(\pi / 4) \int d x|x, 0\rangle \propto \int d x|x, x\rangle .
$$

The outgoing EPR state [14], a two-mode momentum eigenstate with total momentum $p_{1}+p_{2}=0$ and relative position $x_{1}-x_{2}=0$, contains exactly the correlations we need for reliable teleportation. It corresponds to a two-mode squeezed state [15], obtained by superimposing a momentum-squeezed and a position-squeezed state, in the limit of infinite squeezing. We use the Heisenberg representation to describe an approximate version of this state for finite squeezing. The beamsplitter operation applied to a momentum-squeezed and a position-squeezed vacuum mode yields for the Heisenberg operators 15

$$
\begin{aligned}
& \hat{x}_{1}=\left(e^{+r_{1}} \hat{x}_{1}^{(0)}+e^{-r_{2}} \hat{x}_{2}^{(0)}\right) / \sqrt{2}, \\
& \hat{p}_{1}=\left(e^{-r_{1}} \hat{p}_{1}^{(0)}+e^{+r_{2}} \hat{p}_{2}^{(0)}\right) / \sqrt{2}, \\
& \hat{x}_{2}=\left(e^{+r_{1}} \hat{x}_{1}^{(0)}-e^{-r_{2}} \hat{x}_{2}^{(0)}\right) / \sqrt{2}, \\
& \hat{p}_{2}=\left(e^{-r_{1}} \hat{p}_{1}^{(0)}-e^{+r_{2}} \hat{p}_{2}^{(0)}\right) / \sqrt{2} .
\end{aligned}
$$

A superscript ' $(0)$ ' denotes initial vacuum modes and $r_{1}, r_{2}$ are the squeezing parameters. For quantum teleportation [3], mode 1 is sent to "Alice" (the sender) and mode 2 is sent to "Bob" (the receiver). Alice's mode is superimposed at a 50/50 beamsplitter with the unknown input mode "in" to be teleported, yielding for 
the relevant quadratures: $\hat{x}_{\mathrm{u}}=\left(\hat{x}_{\mathrm{in}}-\hat{x}_{1}\right) / \sqrt{2}, \hat{p}_{\mathrm{v}}=$ $\left(\hat{p}_{\text {in }}+\hat{p}_{1}\right) / \sqrt{2}$. Alice measures certain classical values $x_{\mathrm{u}}$ and $p_{\mathrm{v}}$ for $\hat{x}_{\mathrm{u}}$ and $\hat{p}_{\mathrm{v}}$. The operators $\hat{x}_{\mathrm{u}}$ and $\hat{p}_{\mathrm{v}}$ collapse in Bob's mode 2 written as $\hat{x}_{2}=\hat{x}_{\text {in }}-\left(\hat{x}_{1}-\hat{x}_{2}\right)-\sqrt{2} \hat{x}_{\mathrm{u}}$, $\hat{p}_{2}=\hat{p}_{\text {in }}+\left(\hat{p}_{1}+\hat{p}_{2}\right)-\sqrt{2} \hat{p}_{\mathrm{v}}$. After receiving Alice's classical results $x_{\mathrm{u}}$ and $p_{\mathrm{v}}$, Bob displaces his mode correspondingly, $\hat{x}_{2} \longrightarrow \hat{x}_{\text {tel }}=\hat{x}_{2}+g \sqrt{2} x_{\mathrm{u}}, \hat{p}_{2} \longrightarrow \hat{p}_{\text {tel }}=$ $\hat{p}_{2}+g \sqrt{2} p_{\mathrm{v}}$. The parameter $g$ describes a normalized gain. For $g=1$, the teleported mode becomes $\hat{x}_{\text {tel }}=\hat{x}_{\text {in }}-\sqrt{2} e^{-r_{2}} \hat{x}_{2}^{(0)}, \hat{p}_{\text {tel }}=\hat{p}_{\text {in }}+\sqrt{2} e^{-r_{1}} \hat{p}_{1}^{(0)}$. Now we assume an arbitrary coherent-state input $\alpha_{\text {in }}=x_{\text {in }}+i p_{\text {in }}$ and calculate the teleportation fidelity, in this case defined by $F \equiv\left\langle\alpha_{\text {in }}\left|\hat{\rho}_{\text {tel }}\right| \alpha_{\text {in }}\right\rangle$ [10]. It describes the overlap between the input and the teleported state $\hat{\rho}_{\text {tel }}$. Up to a factor $\pi$, this fidelity is related to the $Q$ function of the teleported mode $\left[F=\pi Q_{\text {tel }}\left(\alpha_{\text {in }}\right)\right]$ :

$$
F=\frac{1}{2 \sqrt{\sigma_{x} \sigma_{p}}} \exp \left[-(1-g)^{2}\left(\frac{x_{\mathrm{in}}^{2}}{2 \sigma_{x}}+\frac{p_{\mathrm{in}}^{2}}{2 \sigma_{p}}\right)\right],
$$

where $\sigma_{x}$ and $\sigma_{p}$ are the variances of the $\mathrm{Q}$ function of the teleported mode for the corresponding quadratures. An average fidelity $F_{\mathrm{av}}>\frac{1}{2}$ (averaged upon the complex plane) is only achievable using entanglement [10]. For $g=1$, in fact, 'classical teleportation' with $r_{1}=r_{2}=0$ yields $F_{\mathrm{av}}=F=\frac{1}{2}$ for a coherent input and $\left\langle\Delta \hat{x}^{2}\right\rangle_{\text {vacuum }}=\left\langle\Delta \hat{p}^{2}\right\rangle_{\text {vacuum }}=\frac{1}{4}$. However, for any $r_{1}>0$ or $r_{2}>0$ we obtain $F>\frac{1}{2}$ with $g=1$. We must conclude that the Gaussian two-mode state obtained by superimposing one single-mode squeezed state with vacuum (any $r_{1}>0, r_{2}=0$ or vice versa) exhibits bipartite entanglement. Of course, reliable teleportation with perfect fidelity $F=1$ (for $g=1$ ) requires $r_{1} \rightarrow \infty$ and $r_{2} \rightarrow \infty$ and hence two single-mode squeezed states superimposed. We set $r_{2}=0$ and look for the maximum fidelity of coherent-state teleportation achievable with one single-mode squeezed state $r_{1}>0$ as entanglement source. For infinite squeezing $r_{1} \rightarrow \infty$ and $r_{2}=0$, we find $F=1 / \sqrt{2}(g=1)$.

Here, a non-classical teleportation fidelity serves as sufficient criterion for the presence of entanglement. Indeed, here, the violation of $F \leq \frac{1}{2}$ in coherent-state teleportation is consistent with the violation of $\left\langle\left(\hat{x}_{1}-\hat{x}_{2}\right)^{2}\right\rangle+\left\langle\left(\hat{p}_{1}+\right.\right.$ $\left.\left.\hat{p}_{2}\right)^{2}\right\rangle \geq 1$ which has been recently identified as a sufficient inseparability criterion for bipartite continuous-variable systems [16]. A simpler but less compelling method than doing quantum teleportation for the experimental application of this sufficient criterion would be the detection of the variances $\left\langle\left(\hat{x}_{1}-\hat{x}_{2}\right)^{2}\right\rangle$ and $\left\langle\left(\hat{p}_{1}+\hat{p}_{2}\right)^{2}\right\rangle$ after combining the two modes at a beamsplitter 17.

We now ask if it is also possible to connect more than two locations via EPR channels that can be used for quantum teleportation. At first we consider three locations represented by "Alice", "Bob" and "Claire". Applying the beamsplitter operations ("tritter" [13])

$$
\hat{T}_{123} \equiv \hat{B}_{23}(\pi / 4) \hat{B}_{12}\left(\cos ^{-1} 1 / \sqrt{3}\right),
$$

to a zero-momentum eigenstate in mode 1 and a pair of zero-position eigenstates in modes 2 and 3 yields $\int d x|x, x, x\rangle$. This GHZ-like state [6] is an eigenstate of total momentum zero with relative positions $x_{i}-x_{j}=0$ $(i, j=1,2,3)$. It obviously exhibits tripartite entanglement. In order to consider finite squeezing, we again employ the Heisenberg representation. The tritter applied to a momentum-squeezed and two position-squeezed vacuum modes yields for the Heisenberg operators

$$
\begin{aligned}
& \hat{x}_{1}=\frac{1}{\sqrt{3}} e^{+r_{1}} \hat{x}_{1}^{(0)}+\sqrt{\frac{2}{3}} e^{-r_{2}} \hat{x}_{2}^{(0)}, \\
& \hat{p}_{1}=\frac{1}{\sqrt{3}} e^{-r_{1}} \hat{p}_{1}^{(0)}+\sqrt{\frac{2}{3}} e^{+r_{2}} \hat{p}_{2}^{(0)}, \\
& \hat{x}_{2}=\frac{1}{\sqrt{3}} e^{+r_{1}} \hat{x}_{1}^{(0)}-\frac{1}{\sqrt{6}} e^{-r_{2}} \hat{x}_{2}^{(0)}+\frac{1}{\sqrt{2}} e^{-r_{3}} \hat{x}_{3}^{(0)}, \\
& \hat{p}_{2}=\frac{1}{\sqrt{3}} e^{-r_{1}} \hat{p}_{1}^{(0)}-\frac{1}{\sqrt{6}} e^{+r_{2}} \hat{p}_{2}^{(0)}+\frac{1}{\sqrt{2}} e^{+r_{3}} \hat{p}_{3}^{(0)}, \\
& \hat{x}_{3}=\frac{1}{\sqrt{3}} e^{+r_{1}} \hat{x}_{1}^{(0)}-\frac{1}{\sqrt{6}} e^{-r_{2}} \hat{x}_{2}^{(0)}-\frac{1}{\sqrt{2}} e^{-r_{3}} \hat{x}_{3}^{(0)}, \\
& \hat{p}_{3}=\frac{1}{\sqrt{3}} e^{-r_{1}} \hat{p}_{1}^{(0)}-\frac{1}{\sqrt{6}} e^{+r_{2}} \hat{p}_{2}^{(0)}-\frac{1}{\sqrt{2}} e^{+r_{3}} \hat{p}_{3}^{(0)},
\end{aligned}
$$

with the three squeezing parameters $r_{1}, r_{2}$ and $r_{3}$. The teleportation protocol involving three participants Alice, Bob and Claire works as follows. Let us send the three modes of Eqs. (6) to Alice, Bob and Claire respectively. Again, Alice wants to teleport an unknown quantum state and couples her mode 1 with the unknown input mode "in": $\hat{x}_{\mathrm{u}}=\left(\hat{x}_{\text {in }}-\hat{x}_{1}\right) / \sqrt{2}, \hat{p}_{\mathrm{v}}=\left(\hat{p}_{\text {in }}+\hat{p}_{1}\right) / \sqrt{2}$. Let us write Bob's mode 2 and Claire's mode 3 as

$$
\begin{aligned}
& \hat{x}_{2}=\hat{x}_{\text {in }}-\left(\hat{x}_{1}-\hat{x}_{2}\right)-\sqrt{2} \hat{x}_{\mathrm{u}}, \\
& \hat{p}_{2}=\hat{p}_{\text {in }}+\left(\hat{p}_{1}+\hat{p}_{2}+g^{(3)} \hat{p}_{3}\right)-\sqrt{2} \hat{p}_{\mathrm{v}}-g^{(3)} \hat{p}_{3}, \\
& \hat{x}_{3}=\hat{x}_{\text {in }}-\left(\hat{x}_{1}-\hat{x}_{3}\right)-\sqrt{2} \hat{x}_{\mathrm{u}}, \\
& \hat{p}_{3}=\hat{p}_{\text {in }}+\left(\hat{p}_{1}+g^{(3)} \hat{p}_{2}+\hat{p}_{3}\right)-\sqrt{2} \hat{p}_{\mathrm{v}}-g^{(3)} \hat{p}_{2},
\end{aligned}
$$

where $g^{(3)}$ is another gain determined later. Again, Alice measures certain classical values $x_{\mathrm{u}}$ and $p_{\mathrm{v}}$ for $\hat{x}_{\mathrm{u}}$ and $\hat{p}_{\mathrm{v}}$. The operators $\hat{x}_{\mathrm{u}}$ and $\hat{p}_{\mathrm{v}}$ in Eqs. (7/) collapse. However, this time Alice sends her classical results $x_{\mathrm{u}}$ and $p_{\mathrm{v}}$ either to Bob or Claire via classical channels. Now either Bob or Claire is able to reconstitute the input state provided that additional classical information is received: Bob needs the result of a momentum-detection by Claire reducing $\hat{p}_{3}$ to $p_{3}$ and Claire needs the result of a momentumdetection by Bob reducing $\hat{p}_{2}$ to $p_{2}$. Assuming that Claire detects her mode 3 and sends the result to Bob, a displacement of Bob's mode $2, \hat{x}_{2} \longrightarrow \hat{x}_{\text {tel }}=\hat{x}_{2}+g \sqrt{2} x_{\mathrm{u}}$, $\hat{p}_{2} \longrightarrow \hat{p}_{\text {tel }}=\hat{p}_{2}+g \sqrt{2} p_{\mathrm{v}}+g^{(3)} p_{3}$, accomplishes the teleportation. For $g=1$, the teleported mode becomes

$$
\begin{aligned}
\hat{x}_{\text {tel }} & =\hat{x}_{\text {in }}-\left(\sqrt{3} e^{-r_{2}} \hat{x}_{2}^{(0)}-e^{-r_{3}} \hat{x}_{3}^{(0)}\right) / \sqrt{2} \\
\hat{p}_{\text {tel }} & =\hat{p}_{\text {in }}+\left(2+g^{(3)}\right) e^{-r_{1}} \hat{p}_{1}^{(0)} / \sqrt{3} \\
& +\left(1-g^{(3)}\right) e^{+r_{2}} \hat{p}_{2}^{(0)} / \sqrt{6}+\left(1-g^{(3)}\right) e^{+r_{3}} \hat{p}_{3}^{(0)} / \sqrt{2} .
\end{aligned}
$$


When $r_{1}=r_{2}=r_{3}=r$, the optimum teleportation fidelity is achieved with $g^{(3)}=\left(e^{+4 r}-1\right) /\left(e^{+4 r}+1 / 2\right)$ and becomes for a coherent-state input with Eqs. (8) according to Eq. (丰) for $g=1\left(F=F_{\text {av }}\right)$

$$
F_{\text {opt }}=\left\{\left[1+e^{-2 r}\right]\left[1+3 /\left(2 e^{+2 r}+e^{-2 r}\right)\right]\right\}^{-1 / 2} .
$$

For $r=0$, we obtain $F_{\text {opt }}=\frac{1}{2}$. Perfect teleportation with fidelity $F_{\text {opt }}=1$ is achieved for infinite squeezing in all three single-mode squeezed states $r \rightarrow \infty\left(g^{(3)}=1\right)$. For any $r>0$, we find $F_{\text {opt }}>\frac{1}{2}$. However, again, $F>\frac{1}{2}$ can even be satisfied using only one single-mode squeezed state and two vacua. In this case $\left(r_{2}=r_{3}=0\right)$, we obtain the optimum fidelity $F_{\mathrm{opt}}=\left[2+6 /\left(1+2 e^{+2 r_{1}}\right)\right]^{-1 / 2}$ with $g^{(3)}=\left(e^{+2 r_{1}}-1\right) /\left(e^{+2 r_{1}}+1 / 2\right)$. Remarkably, still $F_{\text {opt }}>\frac{1}{2}$ for any $r_{1}>0$. If Alice and Bob arrange to teleport Alice's unknown coherent state to Claire (and both send the required classical information to Claire and Claire performs the corresponding displacements), one can easily see that with optimum gain the fidelity also exceeds the classical limit for any $r_{1}>0$ when $r_{2}=r_{3}=0$. In fact, Alice, Bob and Claire can determine any one of them as sender and another one as receiver. For $r_{1}, r_{2}, r_{3} \rightarrow \infty$ and unit gain, quantum teleportation is perfect with $F=1$. If $r_{1}=r_{2}=r_{3}=r$, coherent-state teleportation with $F>\frac{1}{2}$ between any two of Alice, Bob and Claire is achieved for any $r>0$, provided optimum gain is used. Even if the tripartite entanglement is based only on one squeezed state, the teleportation is better than classical with any sender and receiver chosen and any nonzero squeezing. For $r_{1} \rightarrow \infty\left(r_{2}=r_{3}=0\right)$, we find the maximum fidelity $F=1 / \sqrt{2}$ as in the scheme involving only Alice and Bob.

In the following we want to investigate if the previous results can be extended to more than three parties. We apply the beamsplitter operations

$$
\begin{aligned}
\hat{N}_{1 \ldots N} \equiv & \hat{B}_{N-1 N}(\pi / 4) \hat{B}_{N-2 N-1}\left(\cos ^{-1} 1 / \sqrt{3}\right) \\
& \times \cdots \times \hat{B}_{12}\left(\cos ^{-1} 1 / \sqrt{N}\right),
\end{aligned}
$$

to a zero-momentum eigenstate in mode 1 and $N-1$ zeroposition eigenstates in modes 2 through $N$. We obtain the entangled $N$-mode state $\int d x|x, x, \ldots, x\rangle$. This state is an eigenstate with total momentum zero and relative positions $x_{i}-x_{j}=0(i, j=1,2, \ldots, N)$.

For finite squeezing, again we refer to the Heisenberg operators. The above " $N$-splitter" applied to one momentum-squeezed $\left(r_{1}=r\right)$ and $N-1$ positionsqueezed vacuum modes $\left(r_{2}=r_{3}=\cdots=r_{N}=r\right)$ yields momentum quadrature operators for any $N$ correlated as

$$
\begin{aligned}
& \left\langle\left(\hat{p}_{k}+\hat{p}_{l}+g^{(N)} \sum_{j \neq k, l}^{N} \hat{p}_{j}\right)^{2}\right\rangle= \\
& \frac{\left[2+(N-2) g^{(N)}\right]^{2}}{4 N} e^{-2 r}+\frac{\left(g^{(N)}-1\right)^{2}(N-2)}{2 N} e^{+2 r},
\end{aligned}
$$

which becomes zero and means perfect correlations for $r \rightarrow \infty$ and $g^{(N)}=1(k \neq l)$. The correlations of the outgoing position quadrature operators satisfy $\left\langle\left(\hat{x}_{k}-\hat{x}_{l}\right)^{2}\right\rangle=e^{-2 r} / 2$. The symmetric $N$-mode Wigner function of these states is a generalization of the finitesqueezing EPR-state Wigner function [18]. With only one momentum-squeezed (squeezing $r_{1}$ ) and $N-1$ vacuum modes $\left(r_{2}=r_{3}=\cdots=r_{N}=0\right)$, the variance in Eq. (11) becomes $\left[2+(N-2) g^{(N)}\right]^{2} e^{-2 r_{1}} /(4 N)+\left(g^{(N)}-\right.$ $1)^{2}(N-2) /(2 N)$ and $\left\langle\left(\hat{x}_{k}-\hat{x}_{l}\right)^{2}\right\rangle=1 / 2$.

Let us now assume that the $N$ outgoing modes are sent to $N$ different locations. We arbitrarily choose two locations of them as sending (mode $k$ ) and receiving station (mode $l$ ) for teleportation. The teleportation protocol is almost the same as in the $N=3$-case. However, now the receiver needs the classical information of the sender's detection of the quadratures $\hat{x}_{\mathrm{u}}=\left(\hat{x}_{\mathrm{in}}-\hat{x}_{k}\right) / \sqrt{2}$, $\hat{p}_{\mathrm{v}}=\left(\hat{p}_{\text {in }}+\hat{p}_{k}\right) / \sqrt{2}$, and in addition the classical results of $N-2$ momentum-detections at the $N-2$ remaining stations. This can be seen by writing mode $l$ as

$$
\begin{aligned}
& \hat{x}_{l}=\hat{x}_{\text {in }}-\left(\hat{x}_{k}-\hat{x}_{l}\right)-\sqrt{2} \hat{x}_{\mathrm{u}}, \\
& \hat{p}_{l}=\hat{p}_{\text {in }}+\hat{p}_{k}+\hat{p}_{l}+g^{(N)} \sum_{j \neq k, l}^{N} \hat{p}_{j}-\sqrt{2} \hat{p}_{\mathrm{v}}-g^{(N)} \sum_{j \neq k, l}^{N} \hat{p}_{j} .
\end{aligned}
$$

Finally, the receiver displaces his mode by the sum of all classical results received, $\hat{x}_{l} \longrightarrow \hat{x}_{\text {tel }}=\hat{x}_{l}+g \sqrt{2} x_{\mathrm{u}}$, $\hat{p}_{l} \longrightarrow \hat{p}_{\text {tel }}=\hat{p}_{l}+g \sqrt{2} p_{\mathrm{v}}+g^{(N)} \sum_{j \neq k, l}^{N} p_{j}$. For $g=1$, the teleported mode becomes $\hat{x}_{\text {tel }}=\hat{x}_{\mathrm{in}}-\left(\hat{x}_{k}-\hat{x}_{l}\right), \hat{p}_{\text {tel }}=$ $\hat{p}_{\text {in }}+\hat{p}_{k}+\hat{p}_{l}+g^{(N)} \sum_{j \neq k, l}^{N} \hat{p}_{j}$.

Now we can optimize the teleportation fidelity using Eq. (11) and find the optimum gain $g^{(N)}=\left[e^{+4 r}-\right.$ $1] /\left[e^{+4 r}+(N-2) / 2\right]$, assuming $r_{1}=r_{2}=\cdots=r_{N}=r$. For a coherent-state input, we obtain the optimum fidelity according to Eq. (何) with $g=1\left(F=F_{\text {av }}\right)$

$$
\begin{aligned}
F_{\mathrm{opt}} & =\left\{1+e^{-2 r}\right\}^{-1 / 2} \\
& \times\left\{1+N /\left[2 e^{+2 r}+(N-2) e^{-2 r}\right]\right\}^{-1 / 2} .
\end{aligned}
$$

For $r=0$, we obtain $F_{\text {opt }}=\frac{1}{2}$. Perfect teleportation with $F_{\text {opt }}=1$ in any of the $N(N-1) / 2$ channels (but, of course, not simultaneously by no-cloning [19]) is achieved with infinite squeezing in all single-mode squeezed states $r \rightarrow \infty\left(g^{(N)}=1\right)$ for any sending and receiving station chosen from the $N$ locations. For any $r>0$, we find $F_{\text {opt }}>\frac{1}{2}$, provided $N \leq 29$. Interestingly, if $N \geq 27$, $F_{\text {opt }}$ reaches a maximum and then drops to a minimum before approaching 1 when the squeezing is increased. For $N \geq 30$, the minimum is below $\frac{1}{2}$, but the maximum (at sufficiently small squeezing) still exceeds $\frac{1}{2}$. Figure 1 shows the fidelity of Eq. (13) for squeezing in $\mathrm{dB}$.

What about using only one single-mode squeezed state and $N-1$ vacua in this $N$-mode scheme? Even in this case $\left(r_{2}=r_{3}=\cdots=r_{N}=0\right)$, quantum teleportation is possible in any of the $N(N-1) / 2$ channels for any $N$. 


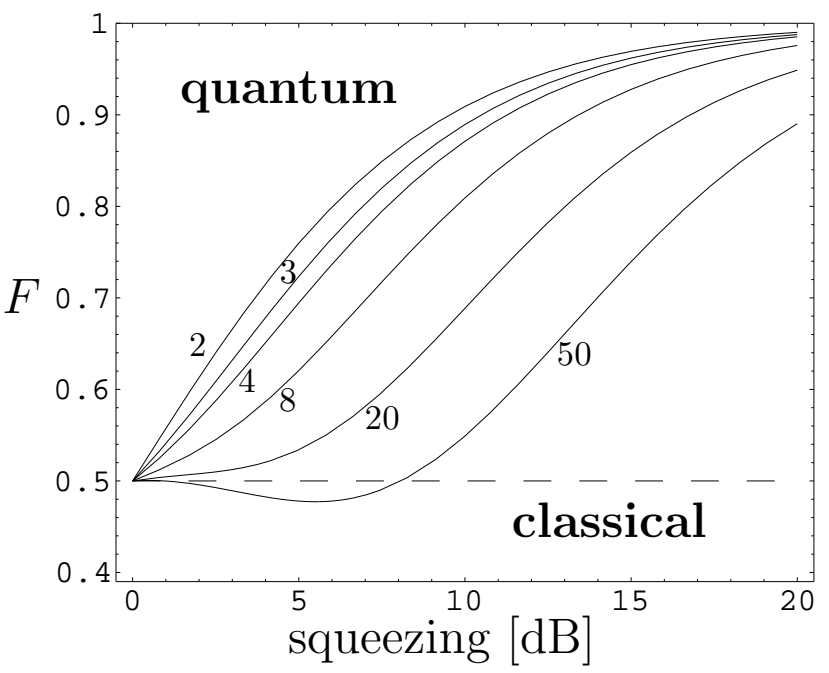

FIG. 1. $N$ squeezed states: Optimized fidelity for the teleportation of an arbitrary coherent state from any sender to any receiver chosen from $N(=2,3,4,8,20$ and 50) parties. A fidelity $F>0.5$ ("quantum") requires $N$-partite entanglement, here produced with $N$ equally squeezed single-mode states. For $N \geq 30$, the fidelity of our protocol becomes classical for some squeezing, but always exceeds 0.5 for sufficiently small squeezing and approaches 1 for infinite squeezing.

We obtain the optimum fidelity for coherent-state teleportation $F_{\text {opt }}=\left[2+2 N /\left(N-2+2 e^{+2 r_{1}}\right)\right]^{-1 / 2}$ with $g^{(N)}=\left[e^{+2 r_{1}}-1\right] /\left[e^{+2 r_{1}}+(N-2) / 2\right]$, shown in Fig. 2 for squeezing in dB. Remarkably, $F_{\text {opt }}>\frac{1}{2}$ for any $r_{1}>0$ with arbitrary $N$. In the limit $r_{1} \rightarrow \infty$, we still attain the maximum fidelity $F=1 / \sqrt{2}$ for any $N$.

By first considering only the momentum-detections at the $N-2$ stations without the teleportation from $k$ to $l$, we can give our protocol also the quality of a 'distillation' of bipartite entanglement from $N$-partite entanglement. The bipartite entanglement can experimentally be verified by applying sufficient inseparability criteria through detections of the combined modes [17 or through quantum teleportation as shown. These verifications require classical communication and local displacements based on the $N-2$ measurement results. However, both in the scheme with $N$ squeezed states and with one squeezed state, mode $k$ and $l$ are projected on bipartite entangled states for any nonzero squeezing and arbitrary $N$ just due to the collapses of the $N-2$ momenta $[18]$. This indicates that in particular our scheme with $N$ squeezed states, yielding classical fidelities for $N \geq 30$ and some squeezing, might not be optimal. Yet asymmetric displacements by the $N-2$ classical results do not provide better fidelities.

In summary, we have considered multipartite entanglement based on quantum variables with a continuous spectrum and a quantum teleportation network using this multipartite entanglement. It can be comparatively easily generated using squeezed light and linear optics.

This work was funded by a DAAD Doktoranden-

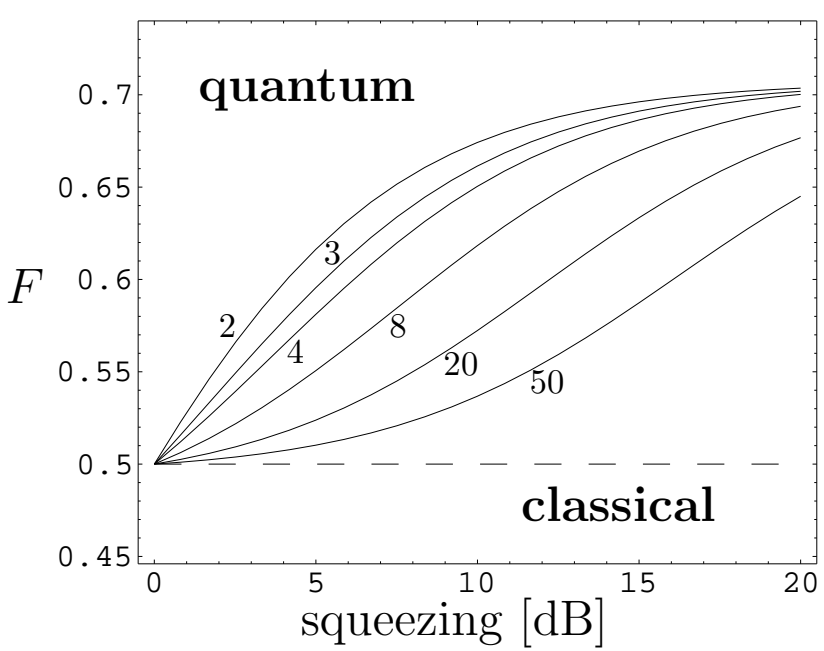

FIG. 2. One squeezed state: Optimized fidelity for the teleportation of an arbitrary coherent state from any sender to any receiver chosen from $N(=2,3,4,8,20$ and 50) parties. A fidelity $F>0.5$ ("quantum") requires $N$-partite entanglement, here produced with one single-mode squeezed state.

stipendium (HSP III) and by the EPSRC Grant No. GR/L91344. SLB thanks Eugene Polzik for suggesting we consider teleportation with non-symmetric squeezing.

[1] C. H. Bennett et al., Phys. Rev. Lett. 70, 1895 (1993).

[2] L. Vaidmann, Phys. Rev. A 49, 1473 (1994).

[3] S. L. Braunstein and H. J. Kimble, Phys. Rev. Lett. 80, 869 (1998).

[4] C. H. Bennett et al., Phys. Rev. A 53, 2046 (1996).

[5] A. V. Thapliyal, Phys. Rev. A 59, 3336 (1999); G. Brassard and T. Mor, Lecture Notes in Computer Science 1509, 1 (1999).

[6] D. M. Greenberger et al., Am. J. Phys. 58, 1131 (1990).

[7] D. Bouwmeester et al., Phys. Rev. Lett. 82, 1345 (1999).

[8] R. Laflamme et al., Phil. Trans. Roy. Soc. Lond. A356, 1941 (1998).

[9] A. Furusawa et al., Science 282, 706 (1998).

[10] S. L. Braunstein, C. A. Fuchs, and H. J. Kimble, to appear in J. Mod. Opt., quant-ph/9910030.

[11] Y. Aharonov et al., Ann. Phys. (NY) 39, 498 (1966).

[12] M. G. A. Paris, Phys. Rev. A 59, 1615 (1999).

[13] S. L. Braunstein, Nature 394, 47 (1998).

[14] A. Einstein, B. Podolsky, and N. Rosen, Phys. Rev. 47, 777 (1935).

[15] D. F. Walls and G. J. Milburn, Quantum Optics, Springer Verlag (1994); special issues on squeezed states, JOSA B 4, 10 (1987); Appl. Phys. B 55, 3 (1992).

[16] L.-M. Duan et al., quant-ph/9908056.

[17] S. M. Tan, Phys. Rev. A 60, 2752 (1999).

[18] P. van Loock and S. L. Braunstein, in preparation.

[19] W. K. Wootters and W. H. Zurek, Nature 299, 802 (1982). 